\title{
LA INSPECCIÓN PEDAGÓGICA EN ÁFRICA: ACTUALIDAD Y POTENCIACIÓN PARA UNA ENSEÑANZA/APRENDIZAJE DE LENGUAS DE CALIDAD
}

\author{
Pedagogical Inspection in Africa: News \\ and empowerment for a teaching / learning \\ of quality languages
}

\author{
Papa Mamour Diop \\ FASTEF/UCAD, Senegal \\ Correo-e: diopmamour2ooo@yahoo.fr \\ Eugénie EYEANG \\ ENS/CRAAL, Gabón \\ Correo-e: eyeangeugenie@gmail.com \\ Recepción: 5 de mayo de 2018 \\ Envío a informantes: 16 de mayo de 2018 \\ Aceptación definitiva: 20 de julio de 2018
}

Resumen: La Inspección pedagógica es una actividad de suma importancia en el proceso de formación de los docentes. Pero pocos son los estudios realizados sobre el tema. En África, hablar de la Inspección pedagógica y del inspector de educación parece tocar un mito. El inspector pedagógico escapa hasta hoy en día a la atención de muchos investigadores del mundo de la educación.

Sucede que la escuela en África se ha regido durante muchos años por normas educativas estrictas procedentes de las entrañas del sistema colonial. Por eso, la figura del controlador o supervisor parece muy vigente, plasmándose además en diferentes cuerpos de inspectores. El objetivo de la presente investigación consiste en presentar el perfil de los inspectores pedagógicos, mostrar sus tareas y esbozar pautas para una mejora de la calidad de la didáctica de las lenguas en África.

Para indagar en los roles del inspector pedagógico, aprovechamos los modelos teóricos de Pérez García (2004) y Poggi et al. (2006) como apoyo para el marco de referencia. A nivel metodológico, este estudio se asienta en el cruce de varios instrumentos de recogida de datos. El método de encuesta mediada por el cuestionario permite recoger las 
representaciones, concepciones y experiencias prácticas de los profesores en activo acerca de la Inspección pedagógica. Los resultados muestran unos desequilibrios que hay que corregir. Los beneficios de esta investigación interesan tanto a los profesionales como a los políticos. A nivel profesional, este análisis debería permitir al inspector centrar más su intervención en la capacitación pedagógica y didáctica de los profesores de lenguas.

Palabras clave: Inspección pedagógica; África; formación docente; didáctica de las lenguas; calidad de la enseñanza.

Aвstract: Pedagogical inspection is a very important activity in the teacher training process. But, few studies are done on the subject. In Africa, to talk about the pedagogical inspection and the inspector of education seems to touch a myth. The pedagogical inspector escapes to the attention of many researchers from the world of education.

It happens that the school in Africa has been governed for many years by strict educational standards from the bowels of the colonial system. For that reason, the figure of the controller or supervisor seems very valid, also being reflected in different bodies of inspectors. The objective of this research is to present the profile of the pedagogical inspectors, show their tasks and outline guidelines for an improvement of the quality of the teaching of languages in Africa.

To investigate the roles of the pedagogical inspector, we took advantage of the theoretical models of Pérez García (2004) and Poggi et al. (2006) as support for the frame of reference. At the methodological level, this study is based on the crossing of several data collection instruments. The survey method mediated by the questionnaire allows to collect the representations, conceptions and practical experiences of the teachers in active about the pedagogical inspection. The results show some imbalances that have to be corrected. The benefits of this research interest both professionals and politicians. At a professional level, this analysis should allow the inspector to focus more on the pedagogical and didactic training of language teachers.

KEY WORDs: Pedagogical inspection; Africa; teacher training; language teaching; quality of teaching.

\section{Introducción general}

$\tau$

A InsPeCción PEDAGógica es una actividad de suma importancia en el proceso de formación de los docentes. Pero pocos son los estudios realizados sobre el tema. En África, hablar de la Inspección pedagógica y del inspector de educación parece tocar a un mito. El inspector pedagógico escapa hasta hoy en día a la atención de muchos investigadores del mundo de la educación.

Los trabajos reseñados procedentes de la Francia metropolitana eran sobre todo vademécums destinados a los aspirantes a la función de inspector de educación y breviarios que describen los roles y funciones encomendados a esta categoría de funcionarios (Vincent, 1967; Voluzan, 1975; Ferrier, 1997) o la naturaleza de la relación entre el inspector y el docente (Paya y Garnier, I97I; Perran, I973).

Posteriormente, la problemática de la Inspección pedagógica ha cristalizado el interés de algunos estudiosos oriundos del contexto africano (Wade, 1986; Faye, 2002; Diop y Faye, 2003; Mengue, 2008). Estos trabajos hacen hincapié globalmente en la ambivalencia de las funciones del inspector pedagógico y la prioridad que se concede 
LA INSPECCIÓN PEDAGÓGICA EN ÁFRICA: ACTUALIDAD Y POTENCIACIÓN PARA UNA ENSEÑANZA/APRENDIZAJE DE LENGUAS DE CALIDAD PAPA MAMOUR DIOP Y EUGÉNIE EYEANG

al control normativo e institucional en detrimento de la evaluación formativa y del asesoramiento pedagógico. Ahora el papel central del inspector en el andamio educativo está siendo objeto de estudio e investigación. Por ello, en base a su acotación en el campo científico-disciplinar de la didáctica de las lenguas, el cometido de la presente investigación consiste en:

- Dar cuenta del perfil, las funciones y responsabilidades de los inspectores pedagógicos en África;

- Identificar las características y el grado de contribución de la Inspección pedagógica en la enseñanza/aprendizaje de lenguas;

- Esbozar pautas de consolidación y reorientación de la Inspección pedagógica con miras a la mejora de la calidad de la didáctica de las lenguas en África.

\section{El marco problemático de la investigación}

Sucede que la escuela en África se ha regido durante muchos años por normas educativas estrictas procedentes de las entrañas del sistema colonial. Por eso, la figura del controlador o supervisor parece muy vigente, plasmándose además en diferentes cuerpos de inspectores. La meta común a estos ejecutivos del aparato educativo consiste en comprobar la adecuación de la práctica docente a las disposiciones institucionales y las orientaciones curriculares (Diop, 2008).

Se comprende pues con mayor profundidad que la formación del profesorado, reproducción del inmovilismo social y del autoritarismo educativo imperante en tiempos de colonización, sea de carácter directivo, en la óptica de un enfoque esencialmente aplicacionista. Hoy en día, una ola de innovación educativa está soplando en todo el continente con la implementación casi generalizada del enfoque por competencias que necesita de un docente de lenguas que Widdowson (1998) y, sobre todo, Vez (2000: 229) definen como:

Un lingüista educativo crítico que media entre teoría y práctica y cuyo perfil podría pasar por la transformación -mediante tareas de formación siguiendo las pautas de investigación-acción crítica- de las teorías del profesorado en auténticas teorías explícitas desde una doble vertiente conceptual y empírica.

En efecto, tal situación redunda con mayor efectividad en el campo de la enseñanza/aprendizaje de las lenguas-culturas que ha experimentado, en los dos últimos decenios, innovaciones tan relevantes como el Marco Común Europeo de Referencia para las Lenguas (MCERL), la promoción del plurilingüismo, la didactización de la interculturalidad, la aplicación de métodos activos basados en el (socio)constructivismo, el enfoque por competencias, etc. Por tanto, la actualización del saber lingüístico, el necesario eclecticismo metodológico y la diferenciación curricular requieren un reciclaje permanente de los profesores de lenguas y la adecuación del dispositivo de enseñanza/aprendizaje a los patrones y estándares internacionales sin perder de vista su imprescindible contextualización. Pues bien, desde sus funciones y responsabilidades, los inspectores africanos especializados en lenguas pueden desempeñar un papel transcendental de asesoramiento y formación de los profesores a y por la práctica; ello está supeditado a una redefinición de la intervención de los inspectores hacia una relación de cooperación profesional y de colaboración pedagógica. 
LA INSPECCIÓN PEDAGÓGICA EN ÁFRICA: ACTUALIDAD Y POTENCIACIÓN PARA UNA ENSEÑANZA/APRENDIZAJE DE LENGUAS DE CALIDAD PAPA MAMOUR DIOP Y EUGÉNIE EYEANG

¿Cómo se caracteriza esta relación desde el perfil, las funciones y responsabilidades de los inspectores?

¿Cuáles son los rasgos descriptivos de la intervención de los inspectores y su impacto en la calidad de la educación en lenguas?

¿Cuáles son los parámetros susceptibles de incidir en la potenciación del actual planteamiento de la Inspección pedagógica en África y, por ende, una enseñanza/ aprendizaje de lenguas de calidad?

Este cuestionamiento ternario concreta la problemática de nuestro estudio y encierra los contenidos que sustentan el tratamiento de la misma.

\section{Marco teórico de la investigación: aproximación histórico-conceptual a la noción de Inspección pedagógica}

Desde un enfoque diacrónico, este apartado revisa la génesis del cuerpo referido a la Inspección pedagógica y la clarificación conceptual de este término tan connotado. En este sentido, es necesario puntualizar de entrada que, en el contexto francófono, fue a raíz de la laicización de la escuela acaecida en Francia en 1903 cuando la idea de vigilancia y control de los establecimientos escolares surgió en los espíritus (Wade, 1986). El incremento de las escuelas y, consecuentemente, del número de maestros avalaron la decisión de editar una Carta de la Enseñanza cuya aplicación era reglada por unos funcionarios que recibían el rebosante título de inspectores de las escuelas. Pero, fue sobre todo en los años 30, es decir, en pleno periodo colonial, cuando la inspección docente adquirió prevalencia. Así pues, en 1934, las instrucciones oficiales estipulaban que:

La misión del inspector de las escuelas primarias consiste en velar por no solo el acatamiento de los estatutos y reglamentos, sino también en asegurarse que los maestros no profesen más que buenas doctrinas y que no den sino buenos ejemplos.

Profesar «buenas doctrinas» releva sin duda alguna de la ideología y se refería tácitamente a la finalidad de la escuela francesa en África; ello no es nada sorprendente en el contexto colonial en que la educación conlleva el proyecto de asimilación de las masas negro-africanas. Por tanto, no es ninguna casualidad que el inspector blanco vea sus prerrogativas reforzadas. Las Instrucciones oficiales ya citadas le encomiendan el siguiente quehacer:

El inspector asegura el mantenimiento de los métodos de enseñanza en la circunscripción principal y en las circunscripciones circundantes; es consultado en cuestiones relacionadas con las adscripciones de puestos, traslados, los galardones del personal docente; las notas de los docentes de la colonia le son comunicadas [...]. Contribuye a la determinación del presupuesto, a la preparación de los pedidos y al reparto del material escolar.

Este enfoque histórico tiene mucho que ver con el ejercicio tradicional de la función de inspector que suscitaba un sentimiento de temor entre los profesores. Lobrot (ı66) abunda en el mismo dictamen:

El docente vive en el terror del inspector. Afirmo y estoy dispuesto a demostrarlo que el $90 \%$ de las actividades de los docentes están condicionadas al temor de verse 
LA INSPECCIÓN PEDAGÓGICA EN ÁFRICA: ACTUALIDAD Y POTENCIACIÓN PARA UNA ENSEÑANZA/APRENDIZAJE DE LENGUAS DE CALIDAD PAPA MAMOUR DIOP Y EUGÉNIE EYEANG

sancionados, juzgados, condenados por un inspector que puede surgir de un momento a otro. Tal reacción que hace sonreír pero que existe, paraliza al docente e impide sus tentativas de buscar métodos eficaces y una relación pedagógica diferente con los alumnos.

Ante la complejidad creciente inherente a la sociedad del conocimiento del siglo XxI, «la rapidez de los cambios y lo imprevisible que caracterizan nuestro mundo (Mayor, 1999), Pérez García (2004: 80) subraya que «históricamente, la inspección ha evolucionado desde una función fiscalizadora y de control hacia otra más centrada en el desarrollo curricular, en la capacitación y en la evaluación formativa». Es decir, aquella visión de rendición de cuentas se desechó por otra donde las relaciones humanas y la sensibilidad por conocer las necesidades eran más importantes, que, finalmente, queda superada por otra que está orientada hacia el fomento del cambio, la motivación y la mejora.

Para construir un marco de referencia para indagar en los roles del inspector pedagógico, aprovechamos los modelos teóricos de Pérez García (2004) y Poggi et al. (2006) que distinguen las siguientes funciones reseñadas en la Tabla I.

TABla I: Reseña de las principales funciones del inspector pedagógico desde los planteamientos teóricos de Pérez García (2004) y Poggi et al. (2006).

\begin{tabular}{|c|c|}
\hline $\begin{array}{c}\text { ROLES DESDE EL MODELO TEÓRICO } \\
\text { DE PÉREZ GARCía }\end{array}$ & $\begin{array}{l}\text { Roles desde EL MODELO PEDAGÓGICO } \\
\text { DE POGGI Y AL. }\end{array}$ \\
\hline $\begin{array}{l}\text { Control y supervisión pedagógicos y organizativos } \\
\text { del funcionamiento del centro. }\end{array}$ & Características de los docentes. \\
\hline Colaboración en la mejora de la práctica docente. & Características de los alumnos. \\
\hline Participación en la evaluación del sistema educativo. & Gestión, organización, comunicación y recursos. \\
\hline $\begin{array}{l}\text { Comprobación del cumplimiento de las leyes, regla- } \\
\text { mentos y disposiciones vigentes. }\end{array}$ & Análisis de la práctica docente. \\
\hline $\begin{array}{l}\text { Asesoramiento, orientación e información en el ejer- } \\
\text { cicio de los derechos y en el cumplimiento de las } \\
\text { obligaciones. }\end{array}$ & Actividad de los alumnos. \\
\hline $\begin{array}{l}\text { Información sobre las actividades de carácter educa- } \\
\text { tivo, promovidas por la Administración, así como so- } \\
\text { bre cualquier aspecto relacionado con la enseñanza. }\end{array}$ & Recurso al currículo formal. \\
\hline
\end{tabular}

Fuente: Pérez García (2004) y Poggi et al. (2006).

Las categorizaciones de García Pérez (2004) y Poggi et al. (2006) ofrecen un marco de análisis de los roles y funciones de los inspectores. Una mirada triangulada de las mismas y su combinación crítica permiten elaborar una parrilla de descripción de las principales funciones cumplidas por los inspectores a partir no solo de las prescripciones, sino también de su propia práctica profesional.

El planteamiento de García Pérez hace hincapié en la prevalencia de las funciones administrativas asignadas a los inspectores mientras que la categorización de Poggi et al. recalca la dimensión pedagógica de su intervención basada en la revisión y explotación de un corpus de informes de inspección. En la taxonomía de García Pérez, la función «colaboración en la mejora de la práctica docente» carece de precisión, si bien se refiere al quehacer pedagógico de los inspectores. A su vez, la clasificación de Poggi, de cariz pedagógico, confirma una actuación centrada en el docente más que en el alumno. Así pues, la atención a las cualidades morales, socioafectivas, cognitivas 
e institucionales del profesor cobra más relevancia que la actividad efectiva de los dicentes enmarcada en un enfoque constructivista del aprendizaje.

\section{Dispositivo contextual y metodológico}

El marco metodológicor de este estudio se asienta en el cruce de varios instrumentos de recogida de datos con el fin de conferir más significatividad y credibilidad a la investigación. Desde esta perspectiva, el método de encuesta mediada por el cuestionario nos permite recoger las representaciones, concepciones y experiencias prácticas de los profesores en activo acerca de la Inspección pedagógica. El cuestionario está administrado a 64 profesores de lenguas titulares de la licenciatura, de la maestría, del máster y del doctorado y repartidos en las Academias educativas de Dakar, Thiès y Saint-Louis. Aparte del encabezado que proporciona datos sobre el encuestado (género, cuerpo docente de adscripción, ancianidad, titulación académico-profesional y lengua extranjera impartida), el cuestionario propiamente dicho consta de 8 preguntas que recaen en las siguientes variables:

- La ocurrencia del contacto con un inspector pedagógico en la carrera profesional;

- El número de encuentros con el inspector;

- La categoría de inspector encontrado;

- El motivo del encuentro con el inspector;

- El enfoque utilizado por el inspector en el transcurso de su actuación;

- Los aspectos más valorados por los inspectores durante su observación-análisis;

- Las funciones del inspector y las expectativas de los profesores de lenguas en cuanto a su intervención;

- Las representaciones de los profesores sobre la calidad de la enseñanza de las lenguas.

Nuestro instrumento de recogida de datos arriba descrito puede distribuirse en dos partes. Las cinco primeras variables se refieren al «Encuentro con el inspector» y facilitan información sobre la frecuencia de los encuentros con el inspector, los objetos y modalidades de los mismos, así como las reacciones emocionales y psicoafectivas del profesor en el momento del contacto con el inspector.

El resto de las variables encajaría en una parte titulada «Roles del inspector y perfil de su intervención». Su meta es de examinar lo que hacen concretamente los inspectores del área de lengua, las expectativas reales de los profesores conforme con las representaciones que tienen del inspector, la orientación de su actuación profesional hacia el control institucional o el acompañamiento pedagógico.

De igual manera, compaginamos el método de encuesta con el análisis de documentos: decretos y ordenanzas, boletines e informes de inspección, para recabar datos sobre el perfil de los inspectores, las misiones se les encomiendan así como las condiciones, normas y resultados de su intervención profesional en el área de Didáctica de las lenguas-culturas extranjeras. En cambio, por constituir el objeto de nuestro estudio a través de su quehacer profesional, los inspectores generales de lenguas no forman parte de la muestra de sujetos implicados en la investigación.

\footnotetext{
Senegal sirve de contexto de encuesta para este estudio.
} 
LA INSPECCIÓN PEDAGÓGICA EN ÁFRICA: ACTUALIDAD Y POTENCIACIÓN PARA UNA ENSEÑANZA/APRENDIZAJE DE LENGUAS DE CALIDAD PAPA MAMOUR DIOP Y EUGÉNIE EYEANG

\subsection{Metodología de análisis de los resultados}

Para una presentación analítica de los datos recogidos, utilizamos el enfoque sistémico de organización del sistema educativo en torno a tres niveles, a saber: el nivel político o macroestructura; el nivel administrativo-institucional o mesoestructura; y el nivel técnico, ejecutivo y didáctico considerado como microestructura. Esta categorización teórica entronca con el planteamiento metodológico de Lahanier Reuter (2018), cuya indagación sobre las innovaciones pedagógicas en el sistema educativo togolés se estructura en tres polos o espacios: el espacio de las prescripciones, el espacio de la formación y el de las prácticas. Pues bien, nuestro dispositivo de análisis de la naturaleza de la Inspección pedagógica se compone de los siguientes niveles subsidiarios unos de otros y que afianzan la complejidad y la transversalidad de todo acto formativo, sea cual sea el área de conocimiento.

Por último, la metodología de análisis de los datos se enmarca en el paradigma cualitativo e interpretativo de investigación y se concreta con la implementación del Análisis de Contenido (Bardin, 1986; Krippendorf, 1990; Negura, 2006) de las comunicaciones escritas, exhumando los niveles manifiesto y latente de las respuestas; sin embargo, algunos parámetros cuantitativos relacionados con la frecuencia, la coocurrencia (Negura, 2006) y la contingencia (Osgood, I979; Bardin, I986) se traslucirán a través del estudio.

\subsection{Presentación y análisis de los datos del trabajo de campo}

Tal y como exponemos en el apartado anterior, la presentación y el análisis de los resultados abarcan los tres espacios de decisión y ordenación del sistema escolar: el espacio de las prescripciones, el espacio de la formación y el de las prácticas docentes.

\section{El espacio de las prescripciones: configuración institucional y misiones de la Inspección pedagógica en África}

En este apartado, acometemos el análisis de los documentos oficiales que rigen la creación, el dispositivo organizativo-funcional y las misiones (y funciones específicas)

TABLA 2: Breve sintesis de las misiones y funciones encomendadas a los inspectores en África

\begin{tabular}{|c|c|c|c|c|}
\hline PAís & ESTRUCTURAS & INSTRUMENTOS LEGALES & MisIONES GENÉRICAS & FUNCIONES ESPECÍFICAS \\
\hline $\begin{array}{l}\text { Costa } \\
\text { de Marfil }\end{array}$ & $\begin{array}{l}\text { Inspección Gene- } \\
\text { ral de la Educa- } \\
\text { ción Nacional }\end{array}$ & $\begin{array}{l}\text { Decreto n. }{ }^{\circ} 72-806 \text { de } \\
\text { Is de diciembre de I972 } \\
\text { (creación) } \\
\text { Circular ministerial } \\
\mathrm{n} .^{\circ} \text { ooI4/MEN/CAB de } \\
28 \text { de febrero de } 200 \mathrm{I} \\
\text { (misiones) } \\
\text { Decreto 2004-564 de } \\
7 \text { de octubre de } 2004 \\
\text { (repartición en dos en- } \\
\text { tidades y actualización } \\
\text { de misiones) }\end{array}$ & $\begin{array}{l}\text { 5. Evaluación de todo } \\
\text { el sistema educativo } \\
\text { 6. Asesoramiento a los } \\
\text { actores y socios del sis- } \\
\text { tema educativo } \\
\text { 7. Información, ani- } \\
\text { mación, formación e } \\
\text { impulso de acciones } \\
\text { implementadas en el } \\
\text { marco de la política } \\
\text { educativa definida a ni- } \\
\text { vel nacional }\end{array}$ & $\begin{array}{l}\text { Presidencia de los tribunales de exá- } \\
\text { menes y oposiciones escolares, peda- } \\
\text { gógicos y profesionales } \\
\text { Vicepresidencia del examen del ba- } \\
\text { chillerato } \\
\text { Presidencia de las comisiones nacio- } \\
\text { nales pedagógicas } \\
\text { Presidencia de la comisión de elec- } \\
\text { ción de las pruebas de exámenes y } \\
\text { oposiciones escolares, profesionales } \\
\text { y pedagógicos }\end{array}$ \\
\hline
\end{tabular}



PAPA MAMOUR DIOP Y EUGÉNIE EYEANG

\begin{tabular}{|c|c|c|c|c|}
\hline País & ESTRUCTURAS & INSTRUMENTOS LEGALES & MisIONES GENÉRICAS & FUNCIONES ESPECÍFICAS \\
\hline Gabón & \begin{tabular}{|l|} 
Inspección Gene- \\
ral de los Servicios
\end{tabular} & $\begin{array}{l}\text { Garantiza la norma } \\
\text { administrativa y peda- } \\
\text { gógica. } \\
\text { No hay un texto legal } \\
\text { sino unos documentos } \\
\text { de trabajo (informes, } \\
\text { protocolos) } \\
\text { Ley n. } .^{\circ} \text { III relativa } \\
\text { al mundo de la educa- } \\
\text { ción en general }\end{array}$ & $\begin{array}{l}\text { Visitas de estableci- } \\
\text { mientos escolares } \\
\text { Control de su buen } \\
\text { funcionamiento } \\
\text { Observación, evalua- } \\
\text { ción, asesoramiento y } \\
\text { formación del personal } \\
\text { docente } \\
\text { Organización de se- } \\
\text { minarios de formación } \\
\text { continuada }\end{array}$ & $\begin{array}{l}\text { Presidencia de tribunales de exáme- } \\
\text { nes y oposiciones escolares, pedagó- } \\
\text { gicos y profesionales } \\
\text { Presidencia de centros del bachille- } \\
\text { rato } \\
\text { Presidencia de las comisiones nacio- } \\
\text { nales pedagógicas } \\
\text { Presidencia de la comisión de elec- } \\
\text { ción de las pruebas de exámenes y } \\
\text { oposiciones escolares, profesionales } \\
\text { y pedagógicos }\end{array}$ \\
\hline R D Congo & $\begin{array}{l}\text { Inspección Ge- } \\
\text { neral de la Ense- } \\
\text { ñanza Primaria, } \\
\text { Secundaria y Pro- } \\
\text { fesional }\end{array}$ & $\begin{array}{l}\text { Ordenanza 9I-23I de I5 } \\
\text { de agosto de I99I }\end{array}$ & $\begin{array}{l}\text { Visita de las escuelas y } \\
\text { control de su buen fun- } \\
\text { cionamiento } \\
\text { Observación, aprecia- } \\
\text { ción, asesoramiento y } \\
\text { formación del personal } \\
\text { docente y control de su } \\
\text { nivel de rendimiento, } \\
\text { Organización de los se- } \\
\text { minarios de formación } \\
\text { continuada }\end{array}$ & $\begin{array}{l}\text { Seguimiento y ejecución de los pro- } \\
\text { gramas e instrucciones oficiales en } \\
\text { materia de enseñanza y educación } \\
\text { escolar } \\
\text { Evaluación de la asimilación de los } \\
\text { programas de las asignaturas y orga- } \\
\text { nización de los tribunales de exáme- } \\
\text { nes y oposiciones } \\
\text { Animación y asesoramiento } \\
\text { Formación permanente } \\
\text { Elaboración de informes sobre los } \\
\text { programas, los manuales y las posi- } \\
\text { bilidades de adaptación para el Mi- } \\
\text { nisterio de Educación } \\
\text { Apreciación de las actividades de los } \\
\text { inspectores y del personal de apoyo }\end{array}$ \\
\hline Senegal & $\begin{array}{l}\text { Inspección Gene- } \\
\text { ral de la Educación } \\
\text { y la Formación }\end{array}$ & \begin{tabular}{|l} 
Decreto 20I4-777 de I2 \\
de junio de 2014
\end{tabular} & $\begin{array}{l}\text { Misión permanente } \\
\text { de seguimiento y eva- } \\
\text { luación de la política } \\
\text { educativa, sobre todo } \\
\text { los tipos de formación, } \\
\text { los programas, los con- } \\
\text { tenidos de enseñanza, } \\
\text { los métodos pedagó- } \\
\text { gicos, los materiales } \\
\text { didácticos, los créditos } \\
\text { horarios, los procedi- } \\
\text { mientos y recursos im- } \\
\text { plementados y la apli- } \\
\text { cación de las reformas } \\
\text { Apoyo de las Inspec- } \\
\text { ciones de Academia } \\
\text { en el pilotaje regional } \\
\text { y desconcentrado, en } \\
\text { particular en la imple- } \\
\text { mentación, la evalua- } \\
\text { ción y el seguimiento } \\
\text { de los contratos de } \\
\text { logros }\end{array}$ & $\begin{array}{l}\text { Promoción de la autoevaluación de } \\
\text { los establecimientos escolares } \\
\text { Publicación periódica de los indica- } \\
\text { dores de calidad y de guías metodo- } \\
\text { lógicas } \\
\text { Explotación y síntesis de los infor- } \\
\text { mes de los inspectores de enseñanza } \\
\text { media y secundaria y de los de la en- } \\
\text { señanza primaria } \\
\text { Evaluación de los exámenes y oposi- } \\
\text { ciones y los currículos de enseñanza } \\
\text { Participación en el control de los } \\
\text { personales de inspección y de direc- } \\
\text { ción y en su contratación } \\
\text { Presidencia de las comisiones encar- } \\
\text { gadas de la reforma de los programas } \\
\text { Participación en la formación conti- } \\
\text { nua a través de seminarios, cursillos, } \\
\text { jornadas de reflexión pedagógica, } \\
\text { por vía de instrucciones oficiales o } \\
\text { publicaciones pedagógicas } \\
\text { Presidencia de los tribunales de certi- } \\
\text { ficación y de selección de profesores } \\
\text { e inspectores de enseñanza media y } \\
\text { secundaria } \\
\text { Validación pedagógica de los ma- } \\
\text { nuales escolares y otros materiales } \\
\text { didácticos }\end{array}$ \\
\hline
\end{tabular}

Fuente: Documentos oficiales consultados. 
LA INSPECCIÓN PEDAGÓGICA EN ÁFRICA: ACTUALIDAD Y POTENCIACIÓN PARA UNA ENSEÑANZA/APRENDIZAJE DE LENGUAS DE CALIDAD PAPA MAMOUR DIOP Y EUGÉNIE EYEANG

de la Inspección pedagógica. Para ello, el siguiente cuadro presenta una breve síntesis de las informaciones clave relacionadas con la Inspección Educativa en dos países del África occidental (Senegal y Costa de Marfil) y dos de África central (Gabón y República Democrática del Congo).

Un análisis detenido del cuadro diseñado permite colegir algunas constancias:

I. Las estructuras de Inspección pedagógica en África se caracterizan por una pluralidad denominativa. Casi todas tienen una vocación general de control y evaluación sobre el sistema educativo y los personales docentes y de dirección encargados de su animación. Por tanto, la variedad terminológica no encierra una diversidad de actuación, pues se nota cierto inmovilismo en cuanto a las misiones y funciones encomendadas; el uso recurrente del lenguaje prescriptivo y directivo lo demuestra a saciedad (control, evaluación, velar por, presidir tribunales, rendimiento, contrato de logros, etc.).

2. Los textos legislativos y reglamentarios, así como los informes de inspección revisados, parecen conceder más trascendencia al control institucional y a la evaluación pedagógica en detrimento de la labor de formación, asesoramiento y creación de dispositivos pedagógicos y metodológicos operativos. Así pues, desde el aparato legal, se espera de los inspectores generales que velen por la debida implementación de las directrices y evalúen los niveles de rendimiento del personal encargado de acatarlas, sin atisbar perspectivas de investigación y elaboración de materiales, métodos y programas eficaces.

Tal planteamiento está corroborado en el discurso de los profesores de lenguas encuestados.

3. El espacio de la formación y de la práctica: rasgos descriptivos y valoración crítica de la contribución de la Inspección pedagógica a la calidad de la enseñanza/ aprendizaje de lenguas en África.

Este apartado recoge y analiza las representaciones, concepciones y propuestas de los profesores de lenguas del contexto educativo de Senegal sobre el personaje del inspector pedagógico y su intervención profesional. La muestra de sujetos implicados nos parece lo suficientemente variada. En efecto, además de cubrir todas las lenguas extranjeras enseñadas en Senegal, incluye a docentes de entre 2 y i6 años de ancianidad, es decir, con un margen de progresión en su trayectoria profesional. Por ejemplo, en función del número de años de servicio activo y de la categorización de Huberman, Thompson y Weiland (2000), situamos la estructura evolutiva de la carrera de dichos profesores en los siguientes tramos: introducción a la carrera (periodo de supervivencia, I-3 años), exploración y estabilización (4-6 años) y experimentación y diversificación (7-I8 años).

Además, los profesores han tenido encuentros con los inspectores con una media de 1,6 veces, habiendo 9 casos de docentes que nunca tuvieron contacto con un inspector de su campo de especialización. Por otra parte, en la mayoría de las veces, se trata de un contacto con inspectores de enseñanza media y secundaria (44 veces) y un inspector general de lengua (II veces). Ello se debe a la escasez de inspectores generales $^{2}$ (63 a nivel nacional, de los cuales 28 son de lenguas). También, la falta de medios

2 Es la misma situación que conocen los docentes en Gabón. Pocos son los docentes que han recibido la visita de un inspector por un acompañamiento pedagógico, sobre todo en el interior del país (EYEANG, 1997). 
logísticos y la lejanía de algunas circunscripciones educativas y centros escolares merman las oportunidades de colaborar con el inspector.

A partir de sus escasos encuentros con los inspectores de lenguas, hemos recogido un conjunto de datos sobre los motivos del contacto, los aspectos más atendidos, el enfoque utilizado por el inspector, las representaciones sobre las funciones actuales y deseadas con miras al incremento de la calidad del proceso de enseñanza/aprendizaje de lenguas.

TABLA 3: Representaciones, concepciones y propuestas de los profesores de lenguas sobre los inspectores y los aspectos valorados durante su actuación

\begin{tabular}{|c|c|c|}
\hline VARIABLES & $\begin{array}{c}\text { RESPUESTAS MÁS DESTACADAS PRESENTADAS POR ORDEN } \\
\text { DE OCURRENCIAS }\end{array}$ & OCURRENCIAS \\
\hline \multirow{5}{*}{$\begin{array}{l}\text { Los motivos del } \\
\text { contacto con el } \\
\text { inspector }\end{array}$} & $\begin{array}{l}\text { Visita de inspección para la certificación de la formación inicial } \\
\text { y para la aspiración a la titularización }\end{array}$ & 42 \\
\hline & Seminarios & II \\
\hline & Animación pedagógica & 05 \\
\hline & Participación en una comisión de examen profesional & 04 \\
\hline & Sesión de la comisión nacional de disciplinas & OI \\
\hline \multirow{6}{*}{$\begin{array}{l}\text { Los aspectos más } \\
\text { valorados por el } \\
\text { inspector }\end{array}$} & $\begin{array}{l}\text { Control de conformidad de la práctica docente con las disposiciones } \\
\text { oficiales (crédito lectivo, programa, enfoque metodológico) }\end{array}$ & $4 \mathrm{I}$ \\
\hline & Respeto de las instrucciones oficiales & 40 \\
\hline & Aspectos deontológicos y de moral profesional & $3 \mathrm{I}$ \\
\hline & Novedades e innovaciones sobre la enseñanza/aprendizaje de la lengua & 06 \\
\hline & Promoción de la innovación y del desarrollo profesional & 05 \\
\hline & Novedades e innovaciones tocante a la lengua enseñada & $\mathrm{O} 3$ \\
\hline \multirow{7}{*}{$\begin{array}{l}\text { Los aspectos } \\
\text { más valorables } \\
\text { para la calidad } \\
\text { de la enseñanza/ } \\
\text { aprendizaje de } \\
\text { lenguas }\end{array}$} & La actualización del programa de lengua & 37 \\
\hline & La contextualización & 34 \\
\hline & $\begin{array}{l}\text { Los manuales y soportes de enseñanza/aprendizaje } \\
\text { La elaboración de manuales y guías metodológicas }\end{array}$ & 32 \\
\hline & La revisión del método de enseñanza/aprendizaje & 27 \\
\hline & $\begin{array}{l}\text { La formación continua y el asesoramiento pedagógico de los } \\
\text { profesores }\end{array}$ & 25 \\
\hline & $\begin{array}{l}\text { El refuerzo lingüístico para un mejor dominio de los contenidos } \\
\text { de enseñanza }\end{array}$ & I3 \\
\hline & $\begin{array}{l}\text { La realización de proyectos didácticos de investigación-acción } \\
\text { implicando a los propios profesores de lenguas }\end{array}$ & 07 \\
\hline
\end{tabular}

Fuente: Encuesta.

Durante los escasos momentos de contacto con el inspector, el propósito del encuentro profesional recae sobre varios objetos, siendo el motivo más frecuente la visita de inspección para la certificación en la formación inicial o en la aspiración a la titularización para profesores interinos en el marco de la formación continua. Cabe destacar que las tareas de formación (seminarios, animación pedagógica, jornada de reflexión didáctica) cobran poco protagonismo y las tareas de investigación en didáctica de lenguas-culturas son inexistentes. Por otro lado, en el acto profesional de intervención, los inspectores tienden a valorar los aspectos administrativos y normativos a expensas del saber y saber hacer referidos al proceso de enseñanza/aprendizaje 
LA INSPECCIÓN PEDAGÓGICA EN ÁFRICA: ACTUALIDAD Y POTENCIACIÓN PARA UNA ENSEÑANZA/APRENDIZAJE DE LENGUAS DE CALIDAD PAPA MAMOUR DIOP Y EUGÉNIE EYEANG

de la lengua impartida. Lo que parecen deplorar los profesores que quieren que los inspectores se dediquen más a la actualización de los programas de lenguas, la elaboración de manuales y materiales didácticos adecuados al contexto educacional de aprendizaje, la formación permanente de los profesores y la investigación-acción sobre problemáticas de índole lingüística y didáctica.

TABLA 4: Representaciones, concepciones y propuestas de los profesores de lenguas sobre los inspectores, sus roles y funciones esenciales

\begin{tabular}{|c|c|c|}
\hline VARIABLE & $\begin{array}{c}\text { RESPUESTAS MÁS DESTACADAS PRESENTADAS } \\
\text { POR ORDEN DE OCURRENCIAS }\end{array}$ & OCURRENCIAS \\
\hline \multirow{3}{*}{$\begin{array}{l}\text { Enfoque } \\
\text { adoptado por el } \\
\text { inspector }\end{array}$} & Colaboración & 39 \\
\hline & Imposición & IO \\
\hline & Sanción & 06 \\
\hline VARIABLE & $\begin{array}{l}\text { ROLES Y FUNCIONES ACTUALES PERCIBIDOS } \\
\text { POR LOS PROFESORES }\end{array}$ & $\begin{array}{l}\text { ROLES Y FUNCIONES DESEADOS } \\
\text { POR LOS PROFESORES }\end{array}$ \\
\hline \multirow{7}{*}{$\begin{array}{l}\text { Roles y } \\
\text { funciones } \\
\text { actuales y } \\
\text { deseados para } \\
\text { la mejora de } \\
\text { la enseñanza/ } \\
\text { aprendizaje de } \\
\text { lenguas }\end{array}$} & Control & \multirow{2}{*}{$\begin{array}{l}\text { Formación y acompañamiento } \\
\text { pedagógico }\end{array}$} \\
\hline & Administración educativa & \\
\hline & Evaluación-sanción & \multirow[t]{2}{*}{ Producción de materiales didáctico } \\
\hline & Evaluación-desarrollo & \\
\hline & \multirow[t]{2}{*}{ Producción de materiales pedagógicos } & Evaluación-desarrollo \\
\hline & & Control \\
\hline & Investigación-acción & Investigación-acción \\
\hline
\end{tabular}

Fuente: Encuesta.

De la tabla diseñada, se desprende que mayoritariamente la percepción del inspector por los profesores ha evolucionado positivamente, pasando de la figura del controlador distante y severo a la de un colaborador y de un asesor pedagógico. Por tanto, se ha derretido la imagen del inspector «colonial» tendente a atemorizar a los docentes. Sin embargo, en base a las representaciones y concepciones de los propios profesores de lenguas, su actuación sigue centrándose en el control institucional y la evaluación pedagógica, ocultando aspectos tan relevantes como la producción de materiales, el acompañamiento pedagógico y la investigación en el área de Didáctica de la lengua.

\section{Discusión y pautas de mejora y potenciación de la contribución de la Inspección pedagógica a la promoción de una enseñanza/aprendizaje de calidad}

En resumidas cuentas, a la luz de los documentos explotados y de la encuesta realizada, la labor profesional de los inspectores reviste un cariz más administrativo, burocrático e institucional que pedagógico-didáctico. Quizá tal situación sea el resultado, en los contextos educativos africanos, del legado del sistema colonial con su concepto de escuela de la autoridad y del constreñimiento. Desde esta perspectiva, los inspectores pedagógicos de lenguas suelen actuar más como controladores que como asesores y acompañadores pedagógicos. También, esta realidad tiene mucho que ver 
en parte con el perfil ${ }^{3}$ de los inspectores generales de lenguas constituidos, en su mayoría, por profesores de universidad adscritos al área de literatura, que suelen presentar carencias en Lingüística y en Didáctica de las lenguas. Lo que puede dar a entender que su dedicación profesional no suele adentrarse en aspectos relacionados con las bases teórico-metodológicas y prácticas de la enseñanza/aprendizaje de las lenguas extranjeras conforme con las aspiraciones de los profesores.

Además, en el ámbito de la enseñanza/aprendizaje de lenguas, la ratio inspector-profesor es muy desorbitada y los medios logísticos muy limitados. Por eso, la Inspección pedagógica suele cubrir solo las zonas urbanas y periurbanas, dejando de lado las circunscripciones educativas provinciales cuyos equipos docentes suelen componerse de profesores con títulos universitarios, pero sin formación pedagógica y didáctica inicial.

Lo susodicho muestra sin ambages la imperiosa necesidad de implementar, de manera concertada y coordinada, una serie de medidas de orden institucional, curricular y didáctico para mejorar el dispositivo de enseñanza/aprendizaje de las lenguas, así como la actuación de cada uno de los elementos del triángulo didáctico (docente, aprendiente y saber).

Desde el punto de vista institucional, el incremento de la calidad de la intervención de los inspectores está supeditado al aumento de los efectivos y de la logística que garantiza su movilidad. La actualización de las leyes y prescripciones hacia la formación, la animación pedagógica, la investigación más que la tradicional inspección fiscalizadora es otro desafío para enmarcar definitivamente la Inspección Educativa en una perspectiva de colaboración y de cooperación.

Desde el lugar de la formación, la contratación de un número suficiente de inspectores generales especializados en Lingüística aplicada, Didáctica de las lenguas y Literatura permitiría un mejor seguimiento de los profesores y el asesoramiento técnico que necesitan para bonificar su intervención en el aula de lengua. Es más, la coordinación de las actividades entre inspectores de enseñanza media y secundaria de lenguas e inspectores generales de lenguas, por una parte, de la formación inicial y de la formación continua, por otra, es imprescindible para la calidad de sendas intervenciones pues, en el terreno, se notan disparidades que desorientan y despistan a los propios profesores. En vez de limitarse a las sempiternas tareas de inspección y de validación de pruebas de exámenes, los inspectores deberían organizar, seminarios de formación, sesiones de animación pedagógica, talleres didácticos y proyectos de investigación sobre la enseñanza/aprendizaje de las lenguas; con lo cual, se podría unir en un «solo proceso docencia, investigación y práctica, caracterizado por el pensar-sentir-haciendo, abriendo espacios de reflexión, compartiendo experiencias, intercambiando inquietudes y cuestionando el hacer profesional» (Rodríguez López, 2004: 610).

Desde el territorio de la práctica, los inspectores generales de lenguas han de indagar más en la enseñanza/aprendizaje de lenguas. Para ello, deben actuar sobre el dispositivo curricular y didáctico: currículos/programas, materiales, métodos y actividades marcados por su carácter rutinario y descontextualizado desde las representaciones y concepciones de los profesores en activo. El cumplimiento de este requisito didáctico es consustancial con la exploración y la investigación del campo disciplinar

Es una situación particular de Senegal. En Gabón, la mayor parte de los inspectores son antiguos profesores de la segundaria que han evolucionado en su carrera profesional por vía de oposiciones y cursillos para obtener el diploma de inspector (EYEANG, 1997). 
LA INSPECCIÓN PEDAGÓGICA EN ÁFRICA: ACTUALIDAD Y POTENCIACIÓN PARA UNA ENSEÑANZA/APRENDIZAJE DE LENGUAS DE CALIDAD PAPA MAMOUR DIOP Y EUGÉNIE EYEANG

de la Didáctica de las lenguas en Senegal y Gabón, como área joven, emergente y en busca de su identidad y de su entidad.

\section{Conclusión}

A raíz del estudio teórico y empírico realizado, se colige que, en África, la Inspección pedagógica todavía no ha logrado superar la clásica visita escolar, cuyo objeto era -tal y como recuerda Pérez García (2004)-, la observación, la comprobación del cumplimiento de la normativa y la redacción de un informe valorativo. Mediante esta investigación acotada al área de la enseñanza/aprendizaje de las lenguas y el cruce de distintos instrumentos de indagación (análisis de documentos y método de encuesta), se desprende que los rasgos de la actuación inspectora se inscriben en el ritualismo académico (y no el pragmatismo pedagógico), el control (y no el asesoramiento), la inspección (y no la visita pedagógica), la evaluación sumativa (en vez de la evaluación formativa) y la retroacción evaluativa (y no la retroacción descriptiva). Por otra parte, la carencia de medios logísticos, el escaso número de la plantilla de inspectores generales y, a veces, su perfil inadecuado a su campo de actuación menoscaban la calidad de su intervención óptima para la mejora del proceso de enseñanza/aprendizaje de lenguas.

No obstante, el interés de esta investigación sobre la Inspección pedagógica en el marco de la Didáctica de las lenguas es innegable. A nivel profesional, este análisis debería permitir al inspector centrar más su intervención en la capacitación pedagógica y didáctica de los profesores de lenguas. En el campo institucional, podría ser una contribución a una mejor adecuación de la selección y de la formación de los inspectores conforme con los patrones y estándares internacionales. Por último, a nivel político, este estudio podría llevar a las autoridades educativas africanas a mejorar las condiciones infraestructurales, materiales e intelectuales de trabajo de los inspectores generales de lenguas con miras a la potenciación de la calidad del dispositivo de enseñanza/aprendizaje.

\section{Bibliografía}

BARdin, L. (1986) Análisis de contenido. Madrid: Akal.

Diop, P. M. y FAYE, L. (2003) Comment l'inspecteur est-il perçu aujourd'bui par les maîtres? Étude circonscrite au département de Thiès. Dossier de recherche en éducation (Fin de formation d'inspecteur de l'enseignement élémentaire). École Normale Supérieure, Université Cheikh Anta Diop de Dakar.

Diop, P. M. (2008) Autonomía y profesionalización de los profesores de lenguas en Senegal: estudio de los actos profesionales docentes desde las estrategias de formación continua. Tesis doctoral. Universidad de Valladolid. Departamento de Didáctica de la Lengua y la Literatura.

EyeANG, E. (1997) L'enseignement/apprentissage de l'espagnol au Gabon. Éléments de recherche pour une adaptation socio-didactique. Université de Grenoble. Thèse à la carte. Villeneuve d'Ascq: Presses universitaires du Septentrion, t. I et 2.

FAYE, B. (2002) Encadrement et perfectionnement des maîtres au Sénégal: les représentations des Inspecteurs et la formation continue par les instituteurs. Mémoire de DEA en Sciences de l'éducation. École Normale Supérieure-Chaire Unesco en Sciences de l'éducation, Université Cheikh Anta Diop de Dakar. 
LA INSPECCIÓN PEDAGÓGICA EN ÁFRICA: ACTUALIDAD Y POTENCIACIÓN PARA UNA ENSEÑANZA/APRENDIZAJE DE LENGUAS DE CALIDAD PAPA MAMOUR DIOP Y EUGÉNIE EYEANG

Ferran, P. (1973) Les Inspecteurs départementaux de l'Éducation Nationale: attributions, style d'autorité. Thèse de Doctorat de $3^{\text {ème }}$ cycle. Université de Paris Sorbonne.

FERRIER, J. (1997) Les inspecteurs des écoles primaires I835-1995, tomes I \& II. Paris: L'Harmattan.

Gabón (20I0) Données mondiales de l'éducation. http://www.ibe.unesco.org/fileadmin/user_ upload/Publications/WDE/20Io/pdf-versions/Gabon.pdf.

García Pérez, M. P. (2004) Inspección. En Francisco Salvador Mata, José Luis Rodríguez Diéguez y Antonio Bolívar Botía (2004) Diccionario Enciclopédico de Didáctica, volumen II, (pp. 8o-8I). Málaga: Ediciones Aljibe.

Guillén Díaz, C. (1997) El área de Didáctica de la Lengua y la Literatura: identidad y entidad de una disciplina especifica. Lenguaje y Textos, I3, II-27.

Hubermann, M.; Thompson, C. y Weiland, S. (2000) Perspectivas de la Carrera del profesor. En B. J. Biddle, T. L. Good y I. F. Goodson (eds.) La enseñanza y los profesores. La profesión de enseñar (pp. 19-98). Barcelona: Paidós.

LaHANier-Reuter, D. (20I8) Quel est le prix de l'innovation? Une étude primaire au Togo. Colloque international sur Innovation à l'école, en classe et dans la formation des enseignants en Afrique du Nord et de l'Ouest, en France et en Allemagne. Mayence (Allemagne), 28-30 juin 2018.

Lовrot, M. (1966) La pédagogie institutionnelle. L'école vers l'autogestion. Paris: Gauthier-Villars.

Mengue, V. (2008) Fonctionnement du suivi pédagogique des enseignants d'EPS au l 'Gabon: cas des communes de Libreville et d'Owendo. Perspectives d'avenir. Mémoire en vue de l'obtention du diplôme d'inspecteur pédagogique. Dakar: InsEPs, Université Cheikh Anta Diop.

Paya, A. J. (I97I) La relation inspecteur-inspecté. Mémoire de Maîtrise. Université de Lyon.

Poggi, M.-P.; Wallian, N. y Musara, M. (2006) La construction sociale du jugement inspectoral: la définition du métier d'enseignant vue à travers une analyse de rapports d'inspection. Revue française de pédagogie, n. ${ }^{\circ} \mathrm{I} 57$, octobre-décembre, I3I-I45.

Présidence de la République Démocratique du Congo (ig9i) Ordonnance $d u$ 9I-23I $d u$ Is aôेt I99I portant règlement d'administration relatif au corps des inspecteurs de l'enseignement primaire, secondaire et professionnel (www.eduquepsp.education/generale/). Consulta el 24/07/I8.

République De Côte D’Ivoire (1972) Décret 72-806 du Is décembre 1972 portant création de l'Inspection Générale de l'Education Nationale (www.menci.igen.net). Consulta el 24/07/18.

République de Côte D'IVOIRe (200I) Arrêté ministériel $n .^{\circ}$ ooI $4 / M E N / C A B d u 28$ février $200 I$ précisant les missions de l'Inspection Générale de l'Éducation Nationale (www.menci.igen. net). Consulta el 24/07/18.

République De CôTe D'Ivolre (2004) Décret 2004-564 du 07 octobre 2004 portant organisation du Ministère de l'Éducation Nationale (www.menci.igen.net). Consulta el 24/07/18.

République Gabonaise (20IO) Loi n. ${ }^{\circ}$ 2I/20II portant orientation générale de l'Éducation, de la Formation et de la Recherche (http://www.unesco.org/education/edurights/media/ docs/25Ic92fb4ei547672cib59748392e6daf2bice5o.pdf). Consulta el I/o9/r8.

RÉPubliQue Du SÉNÉGAL (20I4) Décret 20I4-777 du I2 de juin 2014 portant création, organisation et fonctionnement de l'Inspection Générale de l'Éducation et la Formation.

Rodríguez López, M. (2004) Taller didáctico. En Francisco Salvador Mata, José Luis Rodríguez Diéguez y Antonio Bolívar Botía (2004) Diccionario Enciclopédico de Didáctica, volumen II, (pp. 6io-6II). Málaga: Ediciones Aljibe.

Vincent, G. (1967) Les professeurs du second degré. Paris: Armand Colin.

Voluzan, J. (1975) L'école primaire jugée. Paris: Larousse.

Wade, I. (1986) L'évolution de la fonction d'inspecteur au Sénégal entre 1900 et 1945 . Thèse de doctorat de $3^{\text {ème }}$ cycle. Université Paris Sorbonne. 